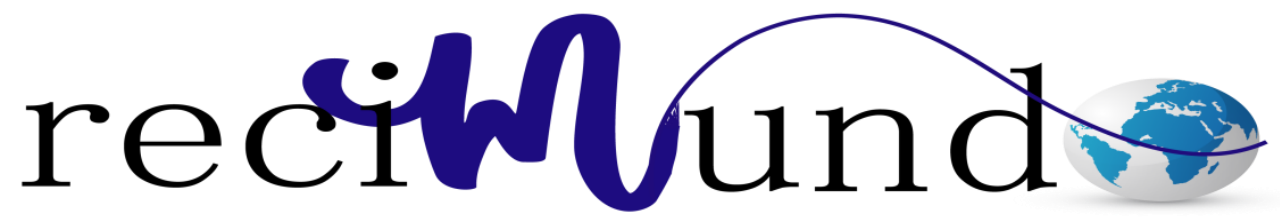

Revista Cientifica Mundo de la Investigación y el Conocimiento

Mónica Fátima Estupiñan Zambrano ${ }^{\text {a. }}$ Paola Geovanna Peñafiel Villareal ${ }^{\text {b. }}$ Gabriela Michelle Andrade Dicao ${ }^{\mathrm{c}}$

Variaciones estacionales de los parámetros físico-químicos de la calidad de agua del estero salado en el canal de acceso al puerto marítimo de Guayaquil bajo condiciones de dragado

Revista Científica Mundo de la Investigación y el Conocimiento. Vol. 2 núm.2, mayo, ISSN: 2588-073X, 2018, pp. 307-326

DOI: $10.26820 /$ recimundo/2.(2).2018.307-326

Editorial Saberes del Conocimiento

Recibido: 05/12/2017 Aceptado: 15/03/2018

a. Universidad Agraria del Ecuador; mestupinan@uagraria.edu.ec

b. Universidad Agraria del Ecuador; ppenafiel@uagraria.edu.ec

c. Universidad Agraria del Ecuador gandrade@uagraria.edu.ec 


\section{Variaciones estacionales de los parámetros físico-químicos de la calidad de agua del estero salado en el canal de acceso al puerto marítimo de Guayaquil bajo condiciones de dragado}

Vol. 2, núm. 2., (2018)

Mónica Fátima Estupiñan Zambrano; Paola Geovanna Peñafiel Villareal; Gabriela Michelle Andrade Dicao

\section{RESUMEN}

El canal de acceso al Puerto Marítimo de Guayaquil, es la vía náutica más importante del país, ya que permite la entrada y salida de embarcaciones de gran calado, incrementando la economía a nivel nacional. Se realizó el estudio en 14 estaciones ubicadas a lo largo del canal en las dos épocas del año húmeda y seca, durante el período 2009-2015.

Las salidas de campo se las efectuó a bordo de una embarcación ligera del Instituto Oceanográfico de la Armada en las estaciones señaladas, siguiendo las instrucciones de campo descritas en los procedimientos del Laboratorio de Oceanografía Química para la recolección de agua estuarina. Se realizaron los análisis físico-químicos, nutrientes y microbiológicos para las muestras recolectadas, de los resultados obtenidos, se elaboraron tablas estadísticas y gráficos de la distribución superficial de los parámetros en estudio.

Los datos adquiridos durante este período, para cada una de las épocas a nivel de superficie, estuvieron dentro de los límites permisibles según la Norma de Calidad Ambiental de la Legislación Secundaria del Ministerio de Ambiente, Libro VI Anexo I (TULSMA) reformado mediante Acuerdo Ministerial No. 097A-2015, aplicado para la preservación de la flora y fauna para aguas dulces, frías o cálidas, y en aguas marinas y de estuario.

Palabras Claves: Calado, agua estuarina, análisis físico-químicos, distribución superficial, límites permisibles. 


\section{Variaciones estacionales de los parámetros físico-químicos de la calidad de agua del estero salado en el canal de acceso al puerto marítimo de Guayaquil \\ bajo condiciones de dragado \\ Vol. 2, núm. 2., (2018) \\ Mónica Fátima Estupiñan Zambrano; Paola Geovanna Peñafiel Villareal; Gabriela Michelle}

Andrade Dicao

\section{ABSTRACT}

The access channel to seaport of Guayaquil, is the country's most important nautical route, allowing the entry and exit of large vessels, increasing the national economy. The study was conducted in fourteen stations located along the channel in both wet and dry seasons of the year during the period 2009-2015.

Field trips were made aboard the slight boat of the INOCAR in designated stations, following the field's instructions procedures described in the Chemical Oceanography Laboratory for collection physical-chemical, microbiological estuarine water for nutrients and collected samples of the results obtained were analyzed and performed on statistical tables and graphs of the surface distribution of the parameters under studies were developed.

The data acquired during this time, for each of the periods at the surface level, were within permissible limits according to the Environmental Quality Standards of Secondary Legislation of the Ministry of Environment, Book VI Annex I ( TULSMA ) reformed by Ministerial Agreement No. 097A -2015, applied for the preservation of the flora and fauna from rivers, cold or warm waters, and marine and estuarine waters .

Key words: Openwork, estuarine water, physical-chemical analysis, surface distribution, permissible limits. 


\section{Variaciones estacionales de los parámetros físico-químicos de la calidad de agua del estero salado en el canal de acceso al puerto marítimo de Guayaquil bajo condiciones de dragado}

Vol. 2, núm. 2., (2018)

Mónica Fátima Estupiñan Zambrano; Paola Geovanna Peñafiel Villareal; Gabriela Michelle Andrade Dicao

\section{Introducción.}

Guayaquil es una ciudad que históricamente ha mantenido una fuerte identidad portuaria, donde se desempeñan las mayores actividades tanto en exportaciones como en importaciones de productos por vía marítima a través de su puerto, que a nivel regional, se ha convertido en el tercero del ranking portuario por su alta competitividad.

La profundidad actual del canal de navegación es de 9.6 m. MLWS (Mean Low Water Spring) y se ha comprobado en los últimos años que el Puerto marítimo de Guayaquil no tiene capacidad de recibir grandes embarcaciones, por lo que paulatinamente perdería competitividad en el contexto internacional. (Falcão \& Vale, 2003)

Es de carácter prioritario que el canal de acceso al Puerto de Guayaquil tenga las mejores características de profundidad a fin de que puedan ingresar barcos de mayores calados y mejores características para transportar grandes volúmenes de carga especialmente de contenedores. Esto constituye un objetivo determinante en el logro de los propósitos finales de convertir a Guayaquil en el centro de la economía nacional, por esta razón se está ejecutando el proyecto del DRAGADO AL CANAL DE ACCESO AL PUERTO MARÍTIMO DE GUAYAQUIL, el mismo que implica la transportación de los sedimentos recolectados en el canal de acceso hacia el área de depósito, lo que genera un intercambio de los parámetros físico químicos en la calidad de las aguas estuarinas, ya que durante el dragado se produce una remoción de los sedimentos de fondo siendo los más livianos los que se suspenden en la columna de agua lo cual podría afectar 


\section{Variaciones estacionales de los parámetros físico-químicos de la calidad de agua del estero salado en el canal de acceso al puerto marítimo de Guayaquil \\ bajo condiciones de dragado}

Vol. 2, núm. 2., (2018)

Mónica Fátima Estupiñan Zambrano; Paola Geovanna Peñafiel Villareal; Gabriela Michelle

Andrade Dicao

a los parámetros de calidad del agua tales como nutrientes, metales pesados, oxígeno disuelto, microbiología y DQO. (Autoridad Portuaria de Guayaquil, 2008)

Debido a las particularidades geográficas del territorio ecuatoriano, existen dos estaciones: húmeda y seca. La estación húmeda o lluviosa se extiende de diciembre a mayo en la costa ecuatoriana con promedio de $148 \mathrm{~mm}$. de precipitación (INOCAR); y la estación seca lo que abarca el resto del año. (LANDAETA, 2010)

El aumento de precipitación en el canal podría provocar alteraciones y afectar la florafauna, salud de la población aledaña. Asimismo los productos resultantes del intercambio en las propiedades físico-químicas y la repartición de contaminantes entre los ecosistemas son arrastrados por las lluvias como precipitados o partículas insolubles.

Por medio de variaciones estacionales, lo que provocan fluctuaciones (movimientos ascendentes y descendentes de la variable) periódicos por naturaleza se pueden manifestar un encadenamiento de intercambio de contaminantes en las columnas de aguas y sedimento. Dicha contaminación es influyente de las actividades antropogénicas ocurridas en años atrás, así como de las descargas de los efluentes de camaroneras y fábricas aledañas a los $60 \mathrm{~km}$. aproximadamente que tiene de longitud el estero salado desde la ciudad de Guayaquil hasta la ciudad de Posorja. Existen ecosistemas estuarinos donde habitan especies de mucha importancia ecológica y comercial, de frágil estabilidad y ardua adaptación a cambios en su hábitat.

Es importante caracterizar los parámetros contaminantes y físico-químicos del canal de acceso al Puerto Marítimo de Guayaquil tanto en época húmeda y seca, en condiciones de 


\section{Variaciones estacionales de los parámetros físico-químicos de la calidad de agua del estero salado en el canal de acceso al puerto marítimo de Guayaquil bajo condiciones de dragado}

Vol. 2, núm. 2., (2018)

Mónica Fátima Estupiñan Zambrano; Paola Geovanna Peñafiel Villareal; Gabriela Michelle Andrade Dicao

dragado ya que es un sector transcendental para el comercio y productividad en la ciudad de Guayaquil.

Este documento presenta el estudio y los cambios que se producen en los parámetros contaminantes y físico-químicos a lo largo del canal de acceso al Puerto Marítimo de Guayaquil en épocas húmedas y secas, durante el período 2009 - 2015.

\section{Metodología.}

De Campo

Se realizaron salidas de campo durante el período 2009-2015 en épocas húmedas y secas.

Los monitoreos se efectuaron en el área de dragado donde se establecieron siete estaciones y, en el área donde se están depositando los sedimentos (producto de dragado) denominada “Área de depósito".

Las salidas de campo durante los años 2014 y 2015, se realizaron en una embarcación con motores fuera de borda del INOCAR.

Se recolectaron muestras de agua a nivel superficial utilizando una botella denominada "Van Dorn", siguiendo el procedimiento interno del Laboratorio de Oceanografía Química del INOCAR.

Las muestras fueron separadas en alícuotas para el análisis de Oxígeno Disuelto, Demanda Química de Oxígeno, Coliformes Fecales, Totales, metales pesados y nutrientes. A 


\section{Variaciones estacionales de los parámetros físico-químicos de la calidad de agua del estero salado en el canal de acceso al puerto marítimo de Guayaquil \\ bajo condiciones de dragado}

Vol. 2, núm. 2., (2018)

Mónica Fátima Estupiñan Zambrano; Paola Geovanna Peñafiel Villareal; Gabriela Michelle

Andrade Dicao

bordo de la embarcación también fueron realizados los análisis in-situ de: Temperatura, pH y salinidad, para lo cual se utilizaron equipos portátiles como un potenciómetro y conductímetro marca WTW, los mismos que estuvieron previamente calibrados con soluciones Buffer certificados Certipur 4, 7, 10 y Estándar de Cloruro de Sodio 35ppm.

Dichas muestras fueron etiquetadas, preservadas en refrigeración y transportadas en coolers hacia los laboratorios del INOCAR y de un laboratorio acreditado.

\section{De Laboratorio}

Las muestras recolectadas en campo, fueron analizadas en el Laboratorio de Oceanografía Química del INOCAR, donde se aplicaron ensayos químicos y metodologías para los parámetros estudiados.

Oxígeno Disuelto

Las muestras para análisis de oxígeno disuelto, son analizadas por volumetría mediante el Procedimiento Específico de Ensayo PEE/LAB-DOQ/01 con un rango de análisis de 0,14 - 9,00 $\mathrm{mg} / \mathrm{l}$, basado en el método yodométrico - modificación de azida del Standard Methods 16st Edition for the examination of water \& wastewater (APHA, 2005).

Demanda Química de Oxígeno

Método PEE-GQM-FQ-04 (procedimiento específico del laboratorio acreditado, para matriz agua). 


\section{Variaciones estacionales de los parámetros físico-químicos de la calidad de agua del estero salado en el canal de acceso al puerto marítimo de Guayaquil bajo condiciones de dragado}

Vol. 2, núm. 2., (2018)

Mónica Fátima Estupiñan Zambrano; Paola Geovanna Peñafiel Villareal; Gabriela Michelle Andrade Dicao

Coliformes Fecales y Totales

Método Tubos Múltiples por Fermentación 9221 B y 9221 E, descritos en el Standard Methods 2005, 21 the edition. Durante la determinación de coliformes totales y fecales, se usaron como control de calidad, blancos de reactivos (medios de cultivo incubado), blanco de medio de dilución (medio de dilución incubado) y blanco de ambiente estéril (Medio de cultivo abierto en el área de trabajo y luego incubado).

Metales Pesados

Método PEE-GQM-F3120B (procedimiento específico del laboratorio acreditado, para matriz agua).

Nitrito

La determinación de este parámetro se basa en una reacción de diazotación, cuando se agrega el ácido sulfanilamida en presencia de nitrito forma una sal de diazonio, la misma que al agregarse $\alpha$-naftilamina, reacción que lleva a la formación de un compuesto azo rosado, cuya intensidad de absorbancia depende del contenido del ion nitrito presente en la muestras, el rango de determinación de este método está entre 0,01 a 2,5 $\mu \mathrm{g}$-at/lt.

Nitrato

El nitrato existente en el agua se reduce casi cuantitativamente a nitrito cuando una muestra es pasada por una columna que contiene limaduras de cadmio cubierta con cobre 


\section{Variaciones estacionales de los parámetros físico-químicos de la calidad de agua del estero salado en el canal de acceso al puerto marítimo de Guayaquil \\ bajo condiciones de dragado}

Vol. 2, núm. 2., (2018)

Mónica Fátima Estupiñan Zambrano; Paola Geovanna Peñafiel Villareal; Gabriela Michelle

Andrade Dicao

metálico. El nitrito producido se determina por diazotación con sulfanilamida y por combinación con N-1 Naftiletilendiamina, para formar un tinte azo fuertemente coloreado cuya absorbancia es proporcional a la cantidad de nitrato inicialmente presente, cuyo rango de detección está entre 0,05 a $45 \mu \mathrm{g}$-at $/ \mathrm{lt}$.

Fosfato

La determinación de este ion, se basa en la reacción del fosfato con molibdato en medio ácido, para formar ácido 1-2-molibdofosfórico y la posterior reducción de éste a un complejo fosfomolibdato de color azul intenso, cuya absorbancia es medida fotométricamente, con un rango determinación entre 0,08 a $6 \mu \mathrm{g}$-at/lt.

\section{Silicato}

Todo los métodos para la determinación de silicato en agua de mar tienen como fundamento la formación de un heteropoliácido por reacción del ácido orto silícico con molibdato en medio ácido, formándose un complejo de color azul como resulta de la reducción del ácido ß-silicomolibdico, para luego ser medido fotométricamente, en un rango de determinación entre 0,1 a $140 \mu \mathrm{g}$-at/lt.

\section{Graficación}

Se realizaron gráficos de distribución a nivel superficial de los parámetros físicoquímicos, microbiológicos, metales pesados y nutrientes respectivamente para cada estación durante los años de estudio. Se emplearon los softwares Excel, ArcGis y Ocean Data View para 


\section{Variaciones estacionales de los parámetros físico-químicos de la calidad de agua del estero salado en el canal de acceso al puerto marítimo de Guayaquil bajo condiciones de dragado}

Vol. 2, núm. 2., (2018)

Mónica Fátima Estupiñan Zambrano; Paola Geovanna Peñafiel Villareal; Gabriela Michelle Andrade Dicao

la elaboración de las imágenes, los que permitieron observar la presencia de algún cambio estacional de dichos parámetros por año.

El procesamiento de los resultados se lo ejecutó por medio del programa ArcGis Map 10.2.2, donde se realizaron gráficos georeferenciados, aplicando métodos estadísticos, zonas de influencia y modelamiento del comportamiento de los parámetros en estudio.

\section{Resultados.}

\begin{tabular}{|c|c|}
\hline \multicolumn{2}{|r|}{ Análisis de los parámetros Físico-químicos } \\
\hline $\begin{array}{c}\text { Temperatura - } \\
\text { Año } 2009\end{array}$ & $\begin{array}{l}\text { Durante la época húmeda del año } 2009 \text {, la temperatura superficial osciló con valores } \\
\text { desde } 27.1^{\circ} \mathrm{C} \text { hasta } 28.4^{\circ} \mathrm{C} \text {, registrados en las Boyas } 33 \text { y } 17 \text { respectivamente, en el } \\
\text { canal de acceso al Puerto Marítimo de Guayaquil, con promedio de } 27.63^{\circ} \mathrm{C} \text {. } \\
\text { Asimismo durante la época seca se registraron valores desde } 25.16^{\circ} \mathrm{C} \text { en la boya } 9 \\
\text { hasta } 27.5^{\circ} \mathrm{C} \text { en la boya } 72 \text {, con promedio de } 26.26^{\circ} \mathrm{C}\end{array}$ \\
\hline $\begin{array}{c}\text { Temperatura - } \\
\text { Año } 2010\end{array}$ & $\begin{array}{l}\text { Los valores registrados de Temperatura en el año } 2010 \text { en época húmeda a nivel } \\
\text { superficial fluctuaron desde } 25.9^{\circ} \mathrm{C} \text { registrado en la Boya } 9 \text {, hasta } 30.7^{\circ} \mathrm{C} \text { en las } \\
\text { boyas } 59 \text { y } 67 \text { con promedio de } 28.27^{\circ} \mathrm{C} \text {. Durante la época seca en dicho año se } \\
\text { reportaron valores de temperatura desde } 24.6^{\circ} \mathrm{C} \text { en la Boya } 33 \text { hasta } 27.2^{\circ} \mathrm{C} \text { en la } \\
\text { Boya } 48 \text {, registrando un promedio de } 25.86^{\circ} \mathrm{C} \text {. }\end{array}$ \\
\hline $\begin{array}{c}\text { Temperatura - } \\
\text { Año } 2011\end{array}$ & $\begin{array}{l}\text { En el año } 2011 \text {, la temperatura a nivel superficial en época húmeda fluctuó desde } \\
25.4^{\circ} \mathrm{C} \text { ubicado en el área de depósito hasta } 27.6^{\circ} \mathrm{C} \text { en las Boyas } 66 \text { y } 67 \text {, con } \\
\text { promedio de } 26.6^{\circ} \mathrm{C} \text {, mientras que en época seca, los valores registrados oscilaron } \\
\text { entre } 26.4^{\circ} \mathrm{C} \text { en la boya } 9 \text { y en el área de depósito, hasta } 28^{\circ} \mathrm{C} \text { en la Boya } 59 \text { con } \\
\text { promedio de } 27.2^{\circ} \mathrm{C} \text {. }\end{array}$ \\
\hline $\begin{array}{c}\text { Temperatura - } \\
\text { Año } 2012\end{array}$ & $\begin{array}{l}\text { Durante la época húmeda del año } 2012 \text {, se registraron valores a nivel superficial de } \\
25.2^{\circ} \mathrm{C} \text { en la Boya } 33 \text { y un máximo de } 26.7^{\circ} \mathrm{C} \text { registrado en la Boya } 9 \text {, con promedio } \\
\text { de } 25.94^{\circ} \mathrm{C} \text {. Asimismo en la época seca se reportaron valores desde } 26.3^{\circ} \mathrm{C} \text { en las } \\
\text { boyas } 9 \text { y } 48 \text {, hasta } 27.2^{\circ} \mathrm{C} \text { ubicado en la Boya } 33 \text { con promedio de } 26.64^{\circ} \mathrm{C} \text {. }\end{array}$ \\
\hline
\end{tabular}




\section{Variaciones estacionales de los parámetros físico-químicos de la calidad de agua del estero salado en el canal de acceso al puerto marítimo de Guayaquil bajo condiciones de dragado}

Vol. 2, núm. 2., (2018) Mónica Fátima Estupiñan Zambrano; Paola Geovanna Peñafiel Villareal; Gabriela Michelle Andrade Dicao

\begin{tabular}{|c|c|}
\hline $\begin{array}{l}\text { Temperatura }- \\
\text { Año } 2013\end{array}$ & $\begin{array}{l}\text { En la época lluviosa del año } 2013 \text {, los valores de temperatura oscilaron desde } 25.1^{\circ} \mathrm{C} \\
\text { ubicado en la Boya } 33 \text {, hasta } 26^{\circ} \mathrm{C} \text { en la Boya } 72 \text {, manifestando un promedio de } \\
25.43^{\circ} \mathrm{C} \text {. Durante la época seca de dicho año, se registraron valores de temperatura } \\
\text { desde } 24.9^{\circ} \mathrm{C} \text { en la Boya } 48 \text { hasta } 27.7^{\circ} \mathrm{C} \text { en la Boya } 72 \text { con promedio de } 25.72^{\circ} \mathrm{C} \text {. }\end{array}$ \\
\hline $\begin{array}{l}\text { Temperatura } \\
\text { Año } 2014\end{array}$ & $\begin{array}{l}\text { En el año } 2014 \text {, se registró en la época húmeda a nivel superficial un máximo valor } \\
\text { de } 30.6^{\circ} \mathrm{C} \text { ubicado en la Boya } 72 \text { y un mínimo de } 28.1^{\circ} \mathrm{C} \text { en el área de depósito de } \\
\text { los sedimentos con un promedio de } 29.52^{\circ} \mathrm{C} \text {. En la época seca se obtuvo un valor } \\
\text { máximo de temperatura superficial de } 27.6^{\circ} \mathrm{C} \text { en la Boya } 59 \text { y un mínimo de } 25.2^{\circ} \mathrm{C} \\
\text { en la estación } 1 \text { del área de depósito, con promedio de } 27.6^{\circ} \mathrm{C} \text {. }\end{array}$ \\
\hline $\begin{array}{l}\text { Temperatura } \\
\text { Año } 2015\end{array}$ & $\begin{array}{l}\text { En el año } 2015 \text {, no se tomaron muestras durante la época húmeda, mientras que en } \\
\text { la seca se registraron valores de temperatura con un máximo de } 30.6^{\circ} \mathrm{C} \text { ubicado en } \\
\text { las Boyas } 33 \text { y } 40 \text { y mínimo de } 29.2^{\circ} \mathrm{C} \text { en la Boya } 8 \text {, manifestando un promedio } \\
\text { general de } 29.9^{\circ} \mathrm{C} \text {. }\end{array}$ \\
\hline $\begin{array}{l}\text { Potencial de } \\
\text { Hidrógeno }- \text { Año } \\
2009\end{array}$ & $\begin{array}{l}\text { Durante la época húmeda del año } 2009 \text {, se reportaron valores de pH desde } 7.69 \mathrm{UpH} \\
\text { registrado en la Boya } 72 \text {, hasta } 8.11 \mathrm{UpH} \text { en el área de depósito, con promedio de } \\
7.95 \mathrm{UpH} \text {. Asimismo en época seca se registraron valores desde } 7.2 \mathrm{UpH} \text { en la } \\
\text { Boya } 72 \text { hasta } 8.29 \mathrm{UpH} \text { en la Boya } 33 \text {, con promedio de } 7.68 \mathrm{UpH} \text {. }\end{array}$ \\
\hline $\begin{array}{l}\text { Potencial de } \\
\text { Hidrógeno }- \text { Año } \\
2010\end{array}$ & $\begin{array}{l}\text { Para la época húmeda del año 2010, se observaron valores de potencial de hidrógeno } \\
\text { con máximo de } 8.33 \mathrm{UpH} \text { en la Boya } 9 \text { y un mínimo de } 7.7 \mathrm{UpH} \text { en las boyas } 67 \text { y } \\
72 \text {, el promedio reportado fue de } 7.98 \mathrm{UpH} \text {. En época seca los valores de pH } \\
\text { oscilaron desde } 8.06 \mathrm{UpH} \text { en la Boya } 72 \text {, hasta } 8.62 \mathrm{UpH} \text { en el área de depósito de } \\
\text { los sedimentos, con promedio de } 8.36 \mathrm{UpH} \text {. }\end{array}$ \\
\hline $\begin{array}{l}\text { Potencial de } \\
\text { Hidrógeno }- \text { Año } \\
2011\end{array}$ & $\begin{array}{l}\text { Los niveles de pH en época húmeda durante el año } 2011 \text { oscilaron entre } 7.75 \mathrm{UpH} \\
\text { localizado en la Boya } 72 \text { hasta } 7.98 \mathrm{UpH} \text { en la Boya } 48 \text {, con promedio de } 7.89 \mathrm{UpH} \\
\text { mientras que en la época seca los niveles de pH registraron un máximo de } 8.06 \mathrm{UpH} \\
\text { en la Boya } 9 \text { y un mínimo de } 7.56 \mathrm{UpH} \text { en la Boya } 72 \text { con promedio de } 7.82 \mathrm{UpH} \text {. }\end{array}$ \\
\hline $\begin{array}{l}\text { Potencial de } \\
\text { Hidrógeno }- \text { Año } \\
2012\end{array}$ & $\begin{array}{l}\text { Durante la época húmeda del año 2012, el potencial de hidrógeno tuvo un máximo } \\
\text { de } 8.79 \mathrm{UpH} \text { en la Boya } 9 \text { y un mínimo de } 8.32 \mathrm{UpH} \text { en la Boya } 72 \text {, con promedio } \\
\text { de } 8.57 \mathrm{UpH} \text {. Asimismo en la época seca los valores de pH fluctuaron desde } 8.2 \\
\text { UpH ubicado en la Boya } 72 \text { hasta } 8.81 \mathrm{UpH} \text { en el área de depósito, con promedio de } \\
\text { 8.56 UpH. }\end{array}$ \\
\hline
\end{tabular}




\section{Variaciones estacionales de los parámetros físico-químicos de la calidad de agua del estero salado en el canal de acceso al puerto marítimo de Guayaquil bajo condiciones de dragado}

Vol. 2, núm. 2., (2018)

Mónica Fátima Estupiñan Zambrano; Paola Geovanna Peñafiel Villareal; Gabriela Michelle Andrade Dicao

\begin{tabular}{|c|c|}
\hline $\begin{array}{l}\text { Potencial de } \\
\text { Hidrógeno }- \text { Año } \\
2013\end{array}$ & $\begin{array}{l}\text { El potencial de Hidrógeno en la época húmeda del año } 2013 \text { registró valores desde } \\
\text { 7.6 UpH en la Boya } 72 \text { hasta } 8.1 \mathrm{UpH} \text { localizado en la Boya } 33 \text {, con promedio de } \\
7.89 \mathrm{UpH} \text {. En época seca los niveles de pH oscilaron desde } 7.3 \mathrm{UpH} \text { a } 8.1 \mathrm{UpH} \\
\text { ubicados en las boyas } 72 \text { y } 17 \text { respectivamente, con promedio de } 7.81 \mathrm{UpH} \text {. }\end{array}$ \\
\hline $\begin{array}{l}\text { Potencial de } \\
\text { Hidrógeno }- \text { Año } \\
2014\end{array}$ & $\begin{array}{l}\text { Para la época húmeda del año } 2014 \text {, el potencial de hidrógeno presentó valores } \\
\text { desde } 7.26 \text { UpH en la boya } 72 \text { hasta } 7.93 \text { UpH en la boya } 17 \text {, con promedio de } 7.74 \\
\text { UpH, mientras que en la época seca se registraron con un máximo de } 8.14 \mathrm{UpH} \text { en la } \\
\text { Boya } 59 \text { y mínimo de } 7.64 \text { en las boyas } 66,67 \text { y } 72 \text { con promedio de } 7.88 \mathrm{UpH} \text {. }\end{array}$ \\
\hline $\begin{array}{l}\text { Potencial de } \\
\text { Hidrógeno }- \text { Año } \\
2015\end{array}$ & $\begin{array}{l}\text { En el año } 2015 \text {, se tomaron muestras en época seca, la misma que registró el nivel } \\
\text { máximo de pH en la estación } 1 \text { del área de depósito con valor de } 7.87 \mathrm{UpH} \text {, mientras } \\
\text { que el mínimo fue de } 7.52 \mathrm{UpH} \text { localizado en la boya } 66 \text {, con promedio de } 7.71 \\
\text { UpH. Durante este año no se realizaron monitoreos en época húmeda. }\end{array}$ \\
\hline $\begin{array}{l}\text { Salinidad - Año } \\
2009\end{array}$ & $\begin{array}{l}\text { Las concentraciones de Salinidad en época seca del año } 2009 \text {, oscilaron entre un } \\
\text { máximo de } 31 \text { UPS ubicado en la boya } 9 \text { y un mínimo de } 20.2 \text { UPS en las boyas } 33 \\
\text { y } 48 \text {, con promedio de } 25.24 \text { UPS. }\end{array}$ \\
\hline $\begin{array}{l}\text { Salinidad - Año } \\
2010\end{array}$ & $\begin{array}{l}\text { Durante la época húmeda se registraron valores que fluctuaron desde } 18.2 \text { hasta } 39.7 \\
\text { UPS ubicados en la boya } 33 \text { y área de depósito respectivamente, con promedio de } \\
22.18 \text { UPS. Asimismo en época seca se observó un máximo de } 46 \text { UPS en el área } \\
\text { de depósito y un mínimo de } 21.1 \text { UPS en la boya } 59 \text {, con promedio de } 28.75 \text { UPS. }\end{array}$ \\
\hline $\begin{array}{l}\text { Salinidad - Año } \\
2011\end{array}$ & $\begin{array}{l}\text { La salinidad registrada en época seca del año } 2011 \text {, presentó valores con un máximo } \\
\text { de } 19.13 \text { UPS y mínimo de } 18.89 \text { UPS, ubicados en las boyas } 66 \text { y } 72 \\
\text { respectivamente. El promedio de los datos registrados fue de 19.04 UPS. }\end{array}$ \\
\hline $\begin{array}{l}\text { Salinidad - Año } \\
2013\end{array}$ & $\begin{array}{l}\text { Para la época seca del año 2013, se registró un máximo de } 26.2 \text { UPS en la Boya } 9 \text { y } \\
\text { mínimo de } 20.1 \text { UPS en la boya 59, con promedio de } 21.69 \text { UPS. } \\
\text { Durante dicho año no se tomaron muestras en época húmeda. }\end{array}$ \\
\hline
\end{tabular}




\section{Variaciones estacionales de los parámetros físico-químicos de la calidad de agua del estero salado en el canal de acceso al puerto marítimo de Guayaquil \\ bajo condiciones de dragado}

Vol. 2, núm. 2., (2018)

Mónica Fátima Estupiñan Zambrano; Paola Geovanna Peñafiel Villareal; Gabriela Michelle

Andrade Dicao

\begin{tabular}{|l|l|}
\hline $\begin{array}{l}\text { Salinidad - Año } \\
\mathbf{2 0 1 4}\end{array}$ & $\begin{array}{l}\text { Durante la época húmeda, se observaron concentraciones de salinidad desde 20.1 } \\
\text { UPS en la Boya 59, hasta 26.2 UPS en la Boya 9, con promedio de 21.69 UPS. } \\
\text { Asimismo en época seca se registró un máximo de 32.3 UPS y mínimo de 21.07 } \\
\text { UPS, ubicados en la estación 6 del área de depósito y la boya 72 respectivamente, } \\
\text { con promedio de 27.67 UPS. }\end{array}$ \\
\hline $\begin{array}{l}\text { Salinidad - Año } \\
\mathbf{2 0 1 5}\end{array}$ & $\begin{array}{l}\text { La salinidad registrada durante la época seca del 2015, presentó valores que oscilan } \\
\text { desde 16.12 UPS en la boya 59, hasta 23.1 UPS en la estación 1 del área de } \\
\text { depósito. El promedio de los datos reportados fue 18.97UPS. }\end{array}$ \\
\hline
\end{tabular}

\begin{tabular}{|c|c|}
\hline \multicolumn{2}{|r|}{ Análisis de los parámetros Químicos } \\
\hline $\begin{array}{l}\text { Oxígeno } \\
\text { Disuelto - Año } \\
2009\end{array}$ & $\begin{array}{l}\text { Las concentraciones de oxígeno disuelto (OD), registradas en época húmeda del año } \\
2009 \text {, oscilaron desde } 6 \mathrm{mg} / \mathrm{l} \text { en la boya } 72 \text { hasta } 7.4 \mathrm{mg} / \mathrm{l} \text { en la boya } 48 \text {, con } \\
\text { promedio de } 6.92 \mathrm{mg} / \mathrm{l} \text {. Asimismo en época seca se reportó un máximo de } 10.6 \mathrm{mg} / 1 \\
\text { y un mínimo de } 7.2 \mathrm{mg} / \mathrm{l} \text {, localizados en las boyas } 9 \text { y } 72 \text { respectivamente, con } \\
\text { promedio de } 8.56 \mathrm{mg} / \mathrm{l} \text {. }\end{array}$ \\
\hline $\begin{array}{l}\text { Oxígeno } \\
\text { Disuelto - Año } \\
2010\end{array}$ & $\begin{array}{l}\text { Para la época húmeda del año 2010, se observó un nivel máximo de OD de } 8.55 \mathrm{mg} / \mathrm{l} \\
\text { ubicado en el área de depósito y un mínimo de } 5.39 \mathrm{mg} / \mathrm{l} \text { en la boya } 72 \text {, con promedio } \\
\text { de } 6.99 \mathrm{mg} / \mathrm{l} \text {, mientras que en época seca se registró un máximo de } 7.75 \mathrm{mg} / \mathrm{l} \text { en la } \\
\text { boya } 17 \text { y un mínimo de } 5.9 \mathrm{mg} / \mathrm{l} \text { en la boya } 72 \text {, con promedio de } 6.87 \mathrm{mg} / \mathrm{l} \text {. }\end{array}$ \\
\hline $\begin{array}{l}\text { Oxígeno } \\
\text { Disuelto - Año } \\
2011\end{array}$ & $\begin{array}{l}\text { Durante la época húmeda del año 2011, se observó la mayor concentración de OD en } \\
\text { la boya } 48 \text { con valor de } 6.81 \mathrm{mg} / \mathrm{l} \text {, la mínima se registró en la boya } 72 \text { con } 5.39 \mathrm{mg} / \mathrm{l} \text { y } \\
\text { promedio de } 6.24 \mathrm{mg} / \mathrm{l} \text {. En época húmeda se reportaron valores desde } 6.03 \mathrm{mg} / \mathrm{l} \mathrm{en} \mathrm{la} \\
\text { boya } 72 \text { hasta } 7.75 \mathrm{mg} / \mathrm{l} \text { en la boya } 17 \text {, con promedio de } 7.05 \mathrm{mg} / \mathrm{l} \text {. }\end{array}$ \\
\hline $\begin{array}{l}\text { Oxígeno } \\
\text { Disuelto - Año } \\
2012\end{array}$ & $\begin{array}{l}\text { Los valores registrados en la época húmeda del año } 2012 \text {, fluctuaron desde } 6.51 \text { hasta } \\
8.32 \mathrm{mg} / \mathrm{l} \text {, localizados en las boyas } 72 \text { y } 48 \text { respectivamente, con promedio de } 7.63 \\
\mathrm{mg} / \mathrm{l} \text {. Durante la época seca se observó un máximo de } 8.38 \mathrm{mg} / \mathrm{l} \text { en la boya } 17 \text {, un } \\
\text { mínimo de } 5.53 \mathrm{mg} / \mathrm{l} \text { en la boya } 72 \text { y promedio de } 7.84 \mathrm{mg} / \mathrm{l} \text {. }\end{array}$ \\
\hline
\end{tabular}




\section{Variaciones estacionales de los parámetros físico-químicos de la calidad de agua del estero salado en el canal de acceso al puerto marítimo de Guayaquil bajo condiciones de dragado}

Vol. 2, núm. 2., (2018)

Mónica Fátima Estupiñan Zambrano; Paola Geovanna Peñafiel Villareal; Gabriela Michelle Andrade Dicao

\begin{tabular}{|c|c|}
\hline $\begin{array}{l}\text { Oxígeno } \\
\text { Disuelto - Año } \\
2013\end{array}$ & $\begin{array}{l}\text { Las concentraciones de OD en época húmeda del año } 2013 \text {, oscilaron desde } 6.3 \mathrm{mg} / \mathrm{l} \\
\text { hasta } 7.96 \mathrm{mg} / \mathrm{l} \text {, localizados en las boyas } 72 \text { y } 9 \text { respectivamente, con promedio de } \\
7.22 \mathrm{mg} / \mathrm{l} \text {, mientras que en época seca se observó una máxima concentración en la } \\
\text { boya } 33 \text { con } 8.02 \mathrm{mg} / \mathrm{l} \text { y una mínima de } 6.16 \mathrm{mg} / \mathrm{l} \text { registrada en la boya } 72 \text {, con } \\
\text { promedio de } 7.20 \mathrm{mg} / \mathrm{l} \text {. }\end{array}$ \\
\hline $\begin{array}{l}\text { Oxígeno } \\
\text { Disuelto - Año } \\
2014\end{array}$ & $\begin{array}{l}\text { Durante la época húmeda se observó una máxima concentración de OD en la boya } 59 \\
\text { con } 7.77 \mathrm{mg} / \mathrm{l} \text { y una mínima de } 5.33 \mathrm{mg} / \mathrm{l} \text { en la boya } 67 \text {, registrando un promedio de } \\
6.33 \mathrm{mg} / \mathrm{l} \text {. En época seca los valores obtenidos de OD oscilaron entre } 2.91 \mathrm{mg} / 1 \\
\text { hasta } 7.83 \mathrm{mg} / \mathrm{l} \text { ubicados en las boyas } 66 \text { y } 33 \text { respectivamente, con promedio de } 4.42 \\
\mathrm{mg} / \mathrm{l} \text {. }\end{array}$ \\
\hline $\begin{array}{l}\text { Oxígeno } \\
\text { Disuelto - Año } \\
2015\end{array}$ & $\begin{array}{l}\text { Se tomaron muestras de OD en época seca del año } 2015 \text {, reportando valores que } \\
\text { fluctúan desde } 4.04 \mathrm{mg} / \mathrm{l} \text { en la boya } 67 \text { hasta } 5.62 \mathrm{mg} / \mathrm{l} \text { en la boya } 33 \text {, con promedio } \\
\text { de } 4.69 \mathrm{mg} / \mathrm{l} \text {. }\end{array}$ \\
\hline $\begin{array}{l}\text { Demanda } \\
\text { Química } \\
\text { Oxígeno }- \text { de } \\
2010\end{array}$ & $\begin{array}{l}\text { Durante la época seca del año } 2010 \text {, se registraron valores de DQO con un máximo de } \\
91.3 \mathrm{mg} / \mathrm{l} \text { en la boya } 72 \text { y un mínimo de } 86.9 \mathrm{mg} / \mathrm{l} \text { ubicado en la boya } 17 \text {. El } \\
\text { promedio de los valores reportados fue de } 87.9 \mathrm{mg} / \mathrm{l}\end{array}$ \\
\hline $\begin{array}{l}\text { Demanda } \\
\text { Química de } \\
\text { Oxígeno - Año } \\
2011\end{array}$ & $\begin{array}{l}\text { En la época seca del año } 2011 \text {, se observó una máxima concentración de DQO en la } \\
\text { boya } 72 \text { con valor de } 91.3 \mathrm{mg} / \mathrm{l} \text {, mientras que la mínima fue registrada en las boyas } 9 \\
\text { y } 17 \text { con } 86.9 \mathrm{mg} / \mathrm{l} \text { y promedio de } 87.79 \mathrm{mg} / \mathrm{l} \text {. }\end{array}$ \\
\hline $\begin{array}{l}\text { Demanda } \\
\text { Química } \\
\text { Oxígeno } \\
2012\end{array}$ & $\begin{array}{l}\text { Los valores reportados de DQO para las épocas húmeda y seca, fueron de } 50 \mathrm{mg} / \mathrm{l} \text { en } \\
\text { todas las estaciones monitoreadas }\end{array}$ \\
\hline $\begin{array}{l}\text { Demanda } \\
\text { Química de } \\
\text { Oxígeno - Año } \\
2013\end{array}$ & $\begin{array}{l}\text { Se registraron en todas las estaciones monitoreadas el valor de } 50 \mathrm{mg} / \mathrm{l} \text {, durante la } \\
\text { época seca del año } 2013 \text {. No se tomaron muestras para la época húmeda en este } \\
\text { período. }\end{array}$ \\
\hline $\begin{array}{l}\text { Demanda } \\
\text { Química } \\
\text { Oxígeno }- \text { Añ } \\
2014\end{array}$ & $\begin{array}{l}\text { Para la época seca del año } 2014 \text {, se observó una mayor concentración de DQO en las } \\
\text { boyas } 17,33,67 \text { y en las estaciones del área de depósito, a excepción de la estación } 2 \text {, } \\
\text { con valor de } 15 \mathrm{mg} / \mathrm{l} \text {, mientras que la mínima fue de } 7 \mathrm{mg} / 1 \text { que se localizó en las } \\
\text { boyas } 48,58,66 \text { y las estaciones } 1,24,6 \text { y } 7 \text { del área de depósito con promedio de } 11 \\
\text { mg/l. }\end{array}$ \\
\hline
\end{tabular}

Análisis de los parámetros Microbiológicos

\begin{tabular}{l|l} 
Coliformes & Para las épocas seca y húmeda del año 2009, las concentraciones de coliformes fecales
\end{tabular} 


\section{Variaciones estacionales de los parámetros físico-químicos de la calidad de agua del estero salado en el canal de acceso al puerto marítimo de Guayaquil bajo condiciones de dragado}

Vol. 2, núm. 2., (2018) Mónica Fátima Estupiñan Zambrano; Paola Geovanna Peñafiel Villareal; Gabriela Michelle Andrade Dicao

\begin{tabular}{|c|c|}
\hline $\begin{array}{c}\text { fecales }-A \tilde{n} o \\
2009\end{array}$ & se mantuvieron uniforme con valor de $2 \mathrm{NMP} / 100 \mathrm{ml}$ en toda el área de estudio. \\
\hline $\begin{array}{l}\text { Coliformes } \\
\text { Fecales - Año } \\
\quad 2014\end{array}$ & $\begin{array}{l}\text { urante la época seca del año 2014, se registró a nivel superficial una máxima } \\
\text { oncentración de Coliformes fecales en la boya 48, con valor de } 32570 \mathrm{NMP} / 100 \mathrm{ml} \text { y } \\
\text { mínima de } 100 \mathrm{NMP} / 100 \mathrm{ml} \text { ubicado en la boya } 66 \text {, con promedio de } 6443.96 \\
\text { IMP/100ml. }\end{array}$ \\
\hline $\begin{array}{l}\text { Coliformes } \\
\text { Fecales - Año } \\
\quad 2015\end{array}$ & $\begin{array}{l}\text { ara la época seca del año en mención, se observó una concentración máxima de } \\
\text { oliformes fecales en la boya } 66 \text { con } 75 \mathrm{NMP} / 100 \mathrm{ml} \text {, mientras que la mínima fue } \\
\text { portada en las boyas } 25,33,40 \text { y en las estaciones } 3,4,5,6 \text { y } 7 \text { del área de depósito } \\
\text { on valor de } 3 \mathrm{NMP} / 100 \mathrm{ml} \text {, registrando un promedio de } 14.28 \mathrm{NMP} / 100 \mathrm{ml} \text {. }\end{array}$ \\
\hline $\begin{array}{l}\text { Coliformes } \\
\text { Totales - Año } \\
\quad 2009\end{array}$ & $\begin{array}{l}\text { as concentraciones de coliformes totales, durante la época húmeda del año } 2009 \text {, } \\
\text { uctuaron desde } 10 \mathrm{NMP} / 100 \mathrm{ml} \text { ubicado en la boya } 59 \text {, hasta } 34 \mathrm{NMP} / 100 \mathrm{ml} \text { en la } \\
\text { oya } 17 \text {, con promedio de } 19.4 \mathrm{NMP} / 100 \mathrm{ml} \text {. Asimismo en la época seca se registraron } \\
\text { alores desde } 2 \mathrm{NMP} / 100 \mathrm{ml} \text { hasta } 34 \mathrm{NMP} / 100 \mathrm{ml} \text {, con promedio de } 10.61 \mathrm{NMP} / 100 \\
\text { l. }\end{array}$ \\
\hline $\begin{array}{r}\text { Colifor } \\
\text { Totales } \\
\mathbf{2 0 1}\end{array}$ & $\begin{array}{l}\text { la época húmeda del año } 2010 \text {, se observó una máxima concentración de } \\
\text { liformes totales en la boya } 59 \text {, con valor de } 1260 \mathrm{NMP} / 100 \mathrm{ml} \text { y la mínima de } 85 \\
\text { MP/100 ml localizado en la boya } 9 \text {, con promedio de } 358.99 \mathrm{NMP} / 100 \mathrm{ml} \text {, mientras } \\
\text { e en época seca las concentraciones de coliformes totales se mantuvieron uniforme } \\
\text { n valor de } 2 \mathrm{NMP} / 100 \mathrm{ml} \text { en toda el área de estudio. }\end{array}$ \\
\hline $\begin{array}{r}\text { Colifo } \\
\text { Totales } \\
201\end{array}$ & $\begin{array}{l}\text { lurante la época húmeda del año } 2012 \text {, se registraron valores de coliformes totales que } \\
\text { scilan desde } 25 \times 10-6 \mathrm{NMP} / 100 \mathrm{ml} \text { en la boya } 9 \text {, hasta } 9.6 \times 10-3 \mathrm{NMP} / 100 \mathrm{ml} \text { en la } \\
\text { oya } 72 \text {, con promedio de } 1.3 \times 10-3 \mathrm{NMP} / 100 \mathrm{ml} \text {. En época seca se observó una } \\
\text { á́xima concentración de este micro-organismo en la boya } 67 \mathrm{con} \text { valor de } 7.2 \times 10-3 \\
\mathrm{JMP} / 100 \mathrm{ml} \text { y mínimo de } 1.9 \times 10-6 \mathrm{NMP} / 100 \mathrm{ml} \text { en la boya } 9 \text {, con promedio de } \\
.1 \times 10-4 \mathrm{NMP} / 100 \mathrm{ml} \text {. }\end{array}$ \\
\hline $\begin{array}{l}\text { Coliformes } \\
\text { Totales - Año } \\
\quad 2013\end{array}$ & rante la época se reportaron datos que van desde $2.5 \times 10-5 \mathrm{NMP} / 100 \mathrm{ml} \mathrm{h}$ \\
\hline $\begin{array}{l}\text { Coliformes } \\
\text { Totales - Año } \\
\quad 2014\end{array}$ & $\begin{array}{l}\text { Las concentraciones de los coliformes totales registrados durante la época húmeda del } \\
\text { año } 2014 \text {, presentaron valores que oscilan desde } 1.4 \times 10-3 \mathrm{NMP} / 100 \mathrm{ml} \text { en la boya } 17 \text {, } \\
\text { hasta } 0.02 \mathrm{NMP} / 100 \mathrm{ml} \text { en la estación } 3 \text { del área de depósito y el promedio de } 4 \times 10-3 \\
\mathrm{NMP} / 100 \mathrm{ml} \text {. Durante la época seca se reportaron concentraciones desde } 100 \\
\mathrm{NMP} / 100 \mathrm{ml} \text {, hasta } 101120 \mathrm{NMP} / 100 \mathrm{ml} \text {, localizados en la estación } 3 \text { del área de } \\
\text { depósito y la boya } 48 \text {, con promedio de } 26893.84 \mathrm{NMP} / 100 \mathrm{ml} \text {. Se puede observar en } \\
\text { la figura } 45 \text {, que la diferencia de concentraciones entre las dos épocas es significante. }\end{array}$ \\
\hline $\begin{array}{l}\text { Coliformes } \\
\text { Totales - Año } \\
\quad 2015\end{array}$ & $\begin{array}{l}\text { ara la época seca del año } 2015 \text {, se observaron concentraciones de este micro- } \\
\text { rganismo desde } 3.6 \mathrm{NMP} / 100 \mathrm{ml} \text {, ubicado en la estación } 1 \text { del área de depósito, hasta } \\
3 \mathrm{NMP} / 100 \mathrm{ml} \text { registrado en la boya } 8 \text {, con promedio de } 37.74 \mathrm{NMP} / 100 \mathrm{ml} \text {. No se } \\
\text { omaron muestras para la época húmeda durante este período. }\end{array}$ \\
\hline
\end{tabular}




\section{Variaciones estacionales de los parámetros físico-químicos de la calidad de agua del estero salado en el canal de acceso al puerto marítimo de Guayaquil bajo condiciones de dragado}

Vol. 2, núm. 2., (2018)

Mónica Fátima Estupiñan Zambrano; Paola Geovanna Peñafiel Villareal; Gabriela Michelle Andrade Dicao

\begin{tabular}{|c|c|}
\hline \multicolumn{2}{|r|}{ Análisis de Metales Pesados } \\
\hline $\begin{array}{c}\text { Plomo }- \text { Año } \\
2009\end{array}$ & $\begin{array}{l}\text { Durante la época húmeda del año } 2009 \text {, este metal registró concentraciones iguales en } \\
\text { toda el área de estudio, con valor de } 0.001 \mathrm{mg} / \mathrm{l} \text {, mientras que en la época seca los datos } \\
\text { fluctuaron desde } 0.001 \mathrm{mg} / \mathrm{l} \text {, hasta } 0.09 \mathrm{mg} / \mathrm{l} \mathrm{en} \mathrm{la} \mathrm{boya} 72 \text {, con promedio de } 0.033 \mathrm{mg} / \mathrm{l} \text {. }\end{array}$ \\
\hline $\begin{array}{l}\text { Plomo - Año } \\
2010\end{array}$ & $\begin{array}{l}\text { Los valores registrados para este parámetro tanto en época seca y húmeda se mantuvieron } \\
\text { uniformes, presentando concentración de } 0.001 \mathrm{mg} / \mathrm{l} \text { en toda el área de estudio. }\end{array}$ \\
\hline $\begin{array}{c}\text { Plomo - Año } \\
2011\end{array}$ & $\begin{array}{l}\text { Durante la época húmeda del año } 2011 \text {, los valores registrados de este metal } \\
\text { permanecieron iguales, con concentración de } 0.01 \mathrm{mg} / \mathrm{l} \text { en todas las estaciones } \\
\text { monitoreadas. Asimismo en la época seca estas concentraciones se mantuvieron } \\
\text { uniformes en toda la zona de estudio con valor de } 0.001 \mathrm{mg} / \mathrm{l} \text {. }\end{array}$ \\
\hline $\begin{array}{c}\text { Plomo - Año } \\
2013\end{array}$ & $\begin{array}{l}\text { Los valores reportados del metal Plomo para la época húmeda del año } 2013 \text {, fueron } \\
\text { iguales en todas las estaciones de estudio registrando una concentración de } 0.2 \mathrm{mg} / \mathrm{l} \text {, sin } \\
\text { embargo en la época seca no se detectaron concentraciones de este parámetro en todo el } \\
\text { canal de acceso. }\end{array}$ \\
\hline $\begin{array}{c}\text { Plomo - Año } \\
2014\end{array}$ & $\begin{array}{l}\text { Las concentraciones de plomo registradas durante la época húmeda del año en mención se } \\
\text { mantuvieron constantes en toda el área de estudio con valor de } 0.2 \mathrm{mg} / \mathrm{l} \text {, mientras que en } \\
\text { la época seca se reportaron datos que fluctuaban dese } 0.0008 \mathrm{mg} / \mathrm{l} \text {, localizados en todas } \\
\text { las boyas y en las estación } 3 \text { del área de depósito hasta } 0.0014 \mathrm{mg} / 1 \text {, con promedio de } \\
0.0011 \mathrm{mg} / \mathrm{l} \text {. }\end{array}$ \\
\hline $\begin{array}{l}\text { Cadmio - } \\
\text { Año } 2009\end{array}$ & $\begin{array}{l}\text { Se registraron valores para este metal, durante la época seca del año } 2009 \text {, los mismos } \\
\text { que oscilaron desde } 0.001 \mathrm{mg} / \mathrm{l} \text { en las boyas } 9,59,66,67,72 \text { y la estación del área de } \\
\text { depósito, hasta un máximo de } 0.06 \mathrm{mg} / \mathrm{l} \text { en la boya } 17, \text { con promedio de } 0.012 \mathrm{mg} / \mathrm{l} \text {. }\end{array}$ \\
\hline $\begin{array}{l}\text { Cadmio - } \\
\text { Año } 2011\end{array}$ & $\begin{array}{l}\text { En la época húmeda del año 2011, se observaron los mismos valores para el metal cadmio } \\
\text { en todas las estaciones de estudio con valor de } 0.01 \mathrm{mg} / \mathrm{l} \text {. No se tomaron muestras de este } \\
\text { parámetro durante la época seca de dicho período. }\end{array}$ \\
\hline $\begin{array}{l}\text { Cadmio - } \\
\text { Año } 2012\end{array}$ & $\begin{array}{l}\text { Las concentraciones de cadmio en las épocas secas y húmedas fueron iguales en toda el } \\
\text { área de estudio, registrando un valor de } 0.001 \mathrm{mg} / \mathrm{l} \text {. }\end{array}$ \\
\hline $\begin{array}{l}\text { Cadmio - } \\
\text { Año } 2014\end{array}$ & $\begin{array}{l}\text { Para la época seca del año } 2014 \text {, se observaron concentraciones de este parámetro desde } \\
0.1316 \mathrm{mg} / \mathrm{l} \text { localizado en la boya } 67 \text { hasta } 0.7048 \mathrm{mg} / \mathrm{l} \text { en la boya } 48 \text {, con promedio de } \\
0.25671 \mathrm{mg} / \mathrm{l} \text {. No se tomaron muestras en la época húmeda de este período. }\end{array}$ \\
\hline $\begin{array}{c}\text { Mercurio - } \\
\text { Año } 2009\end{array}$ & $\begin{array}{l}\text { Durante la época seca del año } 2009 \text {, se registraron las mismas concentraciones de } \\
\text { mercurio en el área de estudio del canal de acceso, con valor de } 0.0001 \mathrm{mg} / \mathrm{l} \text {. No se } \\
\text { tomaron muestras durante la época húmeda de dicho período. }\end{array}$ \\
\hline $\begin{array}{c}\text { Mercurio - } \\
\text { Año } 2011\end{array}$ & $\begin{array}{l}\text { Las concentraciones de mercurio reportadas durante la época húmeda del año } 2011 \\
\text { fueron iguales en todas las áreas monitoreadas, con valor de } 0.0001 \mathrm{mg} / \mathrm{l} \text {. No se } \\
\text { realizaron toma de muestras para la época seca del año mencionado. }\end{array}$ \\
\hline $\begin{array}{l}\text { Mercurio - } \\
\text { Año 2012 }\end{array}$ & $\begin{array}{l}\text { Los valores registrados de mercurio en las épocas húmeda y seca del año } 2012 \text { fueron las } \\
\text { mismas en todas las estaciones reportando datos casi nulos con valores de } 0.0001 \mathrm{mg} / \mathrm{l} \text {. }\end{array}$ \\
\hline $\begin{array}{c}\text { Mercurio - } \\
\text { Año } 2014\end{array}$ & $\begin{array}{l}\text { Durante la época seca del año } 2014 \text {, se observaron concentraciones de este metal que } \\
\text { fluctuaron desde } 0.0004 \mathrm{mg} / \mathrm{l} \text { localizado en la boya } 59 \text { y la estación } 1 \text { del área de depósito } \\
\text { hasta un valor máximo de } 0.0609 \mathrm{mg} / \mathrm{l} \text { registrado en la estación } 3 \text { del área de depósito, } \\
\text { con promedio de } 0.02511 \mathrm{mg} / \mathrm{l} \text {. }\end{array}$ \\
\hline $\begin{array}{c}\text { Níquel - Año } \\
2009\end{array}$ & $\begin{array}{l}\text { Las concentraciones del metal níquel presentó el mismo comportamiento en toda el área } \\
\text { de estudio, durante la época seca en el año } 2009 \text {. El valor reportado para este metal es de } \\
0.01 \mathrm{mg} / \mathrm{l} \text {. }\end{array}$ \\
\hline
\end{tabular}

Revista Científica Mundo de la Investigación y el Conocimiento. 2 (2). pp. 307-326 


\section{Variaciones estacionales de los parámetros físico-químicos de la calidad de agua del estero salado en el canal de acceso al puerto marítimo de Guayaquil \\ bajo condiciones de dragado}

Vol. 2, núm. 2., (2018)

Mónica Fátima Estupiñan Zambrano; Paola Geovanna Peñafiel Villareal; Gabriela Michelle

Andrade Dicao

\begin{tabular}{|c|c|}
\hline $\begin{array}{l}\text { Níquel - Año } \\
\quad 2011\end{array}$ & $\begin{array}{l}\text { Para la época húmeda del año } 2011 \text {, los valores registrados en toda la zona estudiada } \\
\text { presentaron la misma concentración de este metal, cuyo valor fue de } 0.1 \mathrm{mg} / \mathrm{l} \text {. }\end{array}$ \\
\hline $\begin{array}{l}\text { Níquel - Año } \\
2012\end{array}$ & $\begin{array}{l}\text { En cuanto a este parámetro, las concentraciones durante las épocas húmeda y seca del año } \\
\text { 2012, se mantuvieron constantes, presentando un valor de } 0.1 \mathrm{mg} / \mathrm{l} \text { en todas las estaciones } \\
\text { de estudio. }\end{array}$ \\
\hline $\begin{array}{l}\text { Níquel - Año } \\
\quad 2014\end{array}$ & $\begin{array}{l}\text { Los niveles de níquel registrados en la época seca del } 2014 \text {, oscilaron desde } 0.0008 \mathrm{mg} / 1 \\
\text { localizado en casi toda el área de estudio a excepción de las estaciones } 1 \text { y } 6 \text { del área de } \\
\text { depósito, hasta } 0.0099 \mathrm{mg} / \mathrm{l} \text { ubicado en la estación } 1 \text { de la zona de depósito de los } \\
\text { sedimentos. El promedio de todos los valores calculados fue de } 0.00171 \mathrm{mg} / \mathrm{l} \text {. }\end{array}$ \\
\hline $\begin{array}{l}\text { Zinc - Año } \\
2009\end{array}$ & $\begin{array}{l}\text { Los valores de Zinc, registrados en la época seca del año en mención, mantuvieron el } \\
\text { mismo nivel en toda la zona de estudio, siendo el mismo de } 0.03 \mathrm{mg} / \mathrm{l} \text {. }\end{array}$ \\
\hline $\begin{array}{l}\text { Zinc - Año } \\
2011\end{array}$ & $\begin{array}{l}\text { A nivel superficial, se registraron concentraciones iguales de Zinc en toda el área } \\
\text { monitoreada, cuyo valor fue de } 0.17 \mathrm{mg} / \mathrm{l} \text { durante la época húmeda del año } 2011 \text {. }\end{array}$ \\
\hline $\begin{array}{l}\text { Zinc-Año } \\
2012\end{array}$ & $\begin{array}{l}\text { Durante el año } 2012 \text {, este metal registró los mismos niveles en todas las estaciones de } \\
\text { muestreo. El valor reportado fue de } 0.17 \mathrm{mg} / \mathrm{l} \text { en las épocas húmeda y seca. }\end{array}$ \\
\hline $\begin{array}{l}\text { Zinc - Año } \\
2014\end{array}$ & $\begin{array}{l}\text { El parámetro zinc, en la estación seca del año } 2014 \text {, presentó valores con un mínimo de } \\
0.0004 \mathrm{mg} / \mathrm{l} \text { registrado en las boyas } 33,48,66,67,72 \text { y en las estaciones } 1,5 \text { del área de } \\
\text { depósito. La máxima concentración se estableció en la boya } 17 \text { con valor de } 0.2966 \mathrm{mg} / \mathrm{l} \text {, } \\
\text { generando un promedio total de } 0.0311 \mathrm{mg} / \mathrm{l} \text {. }\end{array}$ \\
\hline $\begin{array}{l}\text { Cobre - Año } \\
2011\end{array}$ & $\begin{array}{l}\text { La concentración de este metal durante la época húmeda del año } 2011 \text { fue invariable en } \\
\text { todas las estaciones de muestreo, presentando un valor de } 0.05 \mathrm{mg} / \mathrm{l} \text {. }\end{array}$ \\
\hline $\begin{array}{l}\text { Cobre - Año } \\
2012\end{array}$ & $\begin{array}{l}\text { En este período, el nivel de cobre observado durante las épocas húmeda y seca, son } \\
\text { constantes en toda el área de estudio, cuya concentración fue de } 0.05 \mathrm{mg} / \mathrm{l} \text {. }\end{array}$ \\
\hline $\begin{array}{l}\text { Cobre }- \text { Año } \\
2014\end{array}$ & $\begin{array}{l}\text { En la época seca del año 2014, las concentraciones de Cobre presentaron una máxima } \\
\text { concentración en la estación } 3 \text { del área de depósito cuyo valor fue de } 0.09327 \mathrm{mg} / \mathrm{l} \text {, } \\
\text { mientras que la mínima se registró en la boya } 66 \text { con } 0.0001 \mathrm{mg} / \mathrm{l} \text {. El promedio resultante } \\
\text { de los valores reportados fue de } 0.0325 \mathrm{mg} / \mathrm{l} \text {. }\end{array}$ \\
\hline
\end{tabular}

\section{Conclusiones.}

- Se concluyó que los parámetros físico-químicos tuvieron una ligera variación para las dos épocas (húmeda y seca) en toda el área de estudio, registrando los mayores valores para la estación húmeda, a excepción de la salinidad, que tuvo su mayor concentración durante la época seca. Esto se debe por la presencia de precipitaciones en las épocas húmedas, lo que genera reducción de sal en aguas de mar y estuarinas.

- Los niveles de $\mathrm{pH}$ reportados durante este período se encuentran dentro del rango permisible de la Norma de Calidad Ambiental de la Legislación Secundaria del 


\section{Variaciones estacionales de los parámetros físico-químicos de la calidad de agua del estero salado en el canal de acceso al puerto marítimo de Guayaquil bajo condiciones de dragado}

Vol. 2, núm. 2., (2018)

Mónica Fátima Estupiñan Zambrano; Paola Geovanna Peñafiel Villareal; Gabriela Michelle Andrade Dicao

Ministerio de Ambiente, Libro VI Anexo I (TULSMA) reformado mediante Acuerdo Ministerial No. 097A-2015, aplicado para la preservación de la flora y fauna para aguas dulces, frías o cálidas, y en aguas marinas y de estuario, cuyo rango es de 6.5 $\mathrm{UpH}$ hasta $9.5 \mathrm{UpH}$. No se registran límites permisibles de temperatura y salinidad en la normativa señalada.

- Los promedios obtenidos de los parámetros químicos (OD y DQO), registraron mayor concentración de oxígeno en las épocas húmedas de los años estudiados, con promedio de $6.90 \mathrm{mg} / \mathrm{l}(\mathrm{OD})$ y $68.95 \mathrm{mg} / \mathrm{l}$ (DQO). Durante las épocas secas estos valores disminuyen ligeramente con $6.44 \mathrm{mg} / \mathrm{l}$ (OD) y $46.75 \mathrm{mg} / \mathrm{l}$ (DQO). Las concentraciones de oxígeno disuelto se encuentran por encima del límite mínimo permisible por la Ley de Calidad Ambiental (AM 097-A), cuyo valor es 5mg/l, a excepción de los valores reportados en el área de depósito durante los años 2014 y 2015.

- Se determinó que los parámetros microbiológicos (Coliformes Fecales y Totales), registraron menores concentraciones durante las épocas húmedas de los años en estudio, con un promedio general de 2 NMP/100 ml (Coliformes Fecales) y 81.15 NMP/100 ml (Coliformes Totales). Para las épocas secas estos valores tuvieron un gran aumento de micro-organismos con 1700.48 NMP/100 ml (Coliformes Fecales) y 5910.67 NMP/100 ml (Coliformes Totales).

- Los niveles de nutrientes (Nitrato, Nitrito y Silicato), tuvieron comportamiento irregular para las dos épocas durante el período 2009-2015. El ion nitrato presentó 


\section{Variaciones estacionales de los parámetros físico-químicos de la calidad de agua del estero salado en el canal de acceso al puerto marítimo de Guayaquil \\ bajo condiciones de dragado}

Vol. 2, núm. 2., (2018)

Mónica Fátima Estupiñan Zambrano; Paola Geovanna Peñafiel Villareal; Gabriela Michelle

Andrade Dicao

mayor concentración durante las épocas secas, con valor de $5.23 \mathrm{mg} / \mathrm{l}$, mientras que el nitrito registró en las épocas húmedas valores de $0.016 \mathrm{mg} / \mathrm{l}$. Para el silicato se realizaron monitoreos sólo en épocas secas, registrando un promedio general de 49.13 $\mathrm{mg} / \mathrm{l}$. Las concentraciones de nitrato, se encuentran dentro del rango permisible de la Norma de Calidad Ambiental (AM 097-A), cuyo valor es de 200 mg/l.

- Referente a los metales pesados, se reflejaron las mayores concentraciones durante las épocas húmedas para el plomo, cobre, níquel y mercurio, con valores de 0.077, 0.05, 0.1 y $0.17 \mathrm{mg} / \mathrm{l}$ respectivamente. Sin embargo, los metales cadmio y mercurio, registraron sus mayores valores en las épocas secas con 0.09 y 0.009 mg/l respectivamente.

- En cuanto a los límites permisibles de los metales pesados, el mercurio y níquel se encuentran por debajo nivel máximo autorizado de acuerdo al AM 097-A, cuyos valores son $0.0001 \mathrm{mg} / 1(\mathrm{Hg})$ y $0.1 \mathrm{mg} / \mathrm{l}(\mathrm{Ni})$, mientras que el plomo $(0.001 \mathrm{mg} / \mathrm{l})$, cadmio $(0.005 \mathrm{mg} / \mathrm{l})$, cobre $(0.005 \mathrm{mg} / \mathrm{l})$ y zinc $(0.015 \mathrm{mg} / \mathrm{l})$, no se encuentran dentro de los rangos establecidos en la norma mencionada.

\section{Bibliografía.}

Autoridad Portuaria de Guayaquil. (2008). ESTUDIO DE IMPACTO AMBIENTAL PARA LOS TRABAJOS DE DRAGADO PERMANENTE DEL CANAL DE ACCESO AL PUERTO MARİTIMO DE LA CIUDAD DE GUAYAQUIL. Guayaquil: APG.

Falcão, M., \& Vale, C. (2003). Nutrient dynamics in a coastal lagoon (Ria Formosa, Portugal): the importance of lagoonsea water exchanges on the biological productivity. Ciencias Marinas, 29(4), 425-433. 
Variaciones estacionales de los parámetros físico-químicos de la calidad de agua del estero salado en el canal de acceso al puerto marítimo de Guayaquil bajo condiciones de dragado

Vol. 2, núm. 2., (2018)

Mónica Fátima Estupiñan Zambrano; Paola Geovanna Peñafiel Villareal; Gabriela Michelle Andrade Dicao

LANDAETA, C. (2010). POTENCIALES IMPACTOS AMBIENTALES GENERADOS POR EL DRAGADO Y LA DESCARGA DEL MATERIAL DRAGADO. Carabobo: Universidad de Carabobo. 